\title{
LOGICAL STABILITY IN GROUP THEORY
}

\author{
J. T. BALDWIN and JAN SAXL
}

(Received 10 February 1975; revised 30 June 1975)

\begin{abstract}
This paper investigates the logical stability of various groups. Theorem 1: If a group $G$ is stable and locally nilpotent then it is solvable. Theorem 2: Every non-Abelian variety of groups is unstable.

Morley (1965) introduced the notion of a totally transcendental complete first order theory; Shelah (1969) generalized this to the notion of a stable first order theory. The study of stable and unstable first order theories has yielded a number of results: e.g., Lachlan (1974), Shelah (1971 a,b). Various mathematicians, including Keisler and Shelah, have suggested the application of these concepts to the classification of groups.

The classification is complete for Abelian groups (Berthier (1973), Eklof and Fisher (1972), Macintyre (1970)). In this article we begin the classification for non-Abelian groups.

In Section 1, we define the logical concepts involved and establish some special properties of these concepts in the context of groups. In Section 2, we classify a number of particular groups. In Section 3, we give an application of the logical concepts to group theory by proving a locally nilpotent stable group is solvable. In Section 4, we investigate incomplete theories of groups and prove every non-Abelian variety contains an unstable group. Finally, we suggest several specific open problems in the area.

Our notation is consistent with that in Shelah (1971 a). Either that work or Sacks (1972) can serve as a logical reference. We would like to acknowledge useful conversations and correspondence with G. Sabbagh. Several of the results in this paper (notably Lemma 2.2, Theorem 2.3, Theorem 2.4, and Theorem 4.1) were obtained independently by Sabbagh (1975 a, b).
\end{abstract}

\section{Section 1.}

In this section we recount for algebraists the principal logical definitions and results used in this work. A thorough investigation of stable theories occurs 
in Shelah (1971 a) and we rely heavily on that work. After listing a few general results from model theory we turn to some specific formulations for applications to algebra, and in particular the theory of groups.

A complete theory of groups is a maximal consistent set of first order sentences in the language of group theory (i.e. with non-logical symbols, $\cdot,-1$ and 1). In particular, for any group $G, T h(G)=\{\varphi \mid G \|=\varphi\}$ (i.e. the set of first order sentences true in $G$ ) is a complete theory. Rosenstein (1973) elucidates the notion of first order property in a group theoretic setting.

If $A$ is a subgroup of $B$ such that every first order formula (possibly with names for some members of $A$ ) which is true in $A$ is true in $B$ then we say $A$ is an elementary submodel of $B(A \leqslant B)$. Let $b_{0} \in B \geqslant A$. Then the type $p$ which $b_{0}$ realizes over $A$ is the set of all formulas $\varphi\left(v_{0}, \bar{a}\right)$ (where $\bar{a}$ is a sequence of elements from $A$ ) such that $B \mid=\varphi\left(b_{0}, \bar{a}\right)$. We denote by $S(A)$ the set of all types realized in some elementary extension $B$ of $A$. Now for each complete theory $T$ define a function from cardinals to cardinals by

$$
K_{T}(\lambda)=\sup \{|S(A)|: A \mid=T \text { and }|A| \leqq \lambda\} .
$$

(This definition differs slightly from that given by Shelah (1969), but it is easy to see that the difference is mainly notational.)

Shelah (1971a) and Keisler (1974) have shown that for any first order theory $T$ the function $K_{T}(\lambda)$ is one of the following six functions.

i) $K_{T}(\kappa)=\kappa\left(T\right.$ is $\kappa_{0}$-stable or totally transcendental.)

ii) $\kappa+2^{\kappa_{0}}(T$ is superstable.)

iii) $\kappa^{\varkappa_{0}}(T$ is stable. $)$

iv) $K_{T}(\kappa)=\operatorname{ded}(\kappa)(T$ is ordered.)

v) $K_{T}(\kappa)=\operatorname{ded}(\kappa)^{\kappa_{0}}$ ( $T$ is multiply-ordered.)

vi) $K_{T}(\kappa)=2^{\kappa}$ ( $T$ has the independence property.)

Thus the question we shall address is: given a group $A$, into which of these six stability classes does $T h(A)$ fall. We will say, e.g., $A$ is stable if $T h(A)$ is stable. We will not deal here with the distinction between iv) ordered and v) multiply ordered. (For further information on this and the definition of the function ded from cardinals to cardinals see Keisler (1974) and Shelah (1971a: §4.) If $T h(A)$ is in classes iv), v) or vi) we say $A$ is unstable. Note that if $G$ is a finite group then $|S(G)|=|G|$ so our interest is primarily in infinite groups.

The following definitions and results of Shelah link the foregoing "global" properties with "local" properties of particular formulas.

Definition. (a) A formula $\varphi(\bar{x}, \bar{y})$ is unstable in $T$, if for every $n \geqq 1, T$ implies the sentence

$$
\varphi_{n}: \exists \bar{w}_{r_{1}}, \cdots, \exists \bar{w}_{\tau_{2} n-1} \underset{\sigma \in 2^{n}}{\wedge} \exists \bar{v}_{\sigma} \wedge \varphi\left(\bar{v}_{i<n}, \bar{w}_{\sigma i}\right)^{\sigma(i)}
$$

where $\tau_{1}, \cdots, \tau_{2} n-1$ enumerate the binary sequences of length less than $n$, 
$\sigma \uparrow i$ denotes the restriction of the sequence $\sigma$ to $i$, and $\varphi(\bar{x}, \bar{y})^{\sigma(i)}$ denotes $\varphi(\bar{x}, \bar{y})$ or $\square \varphi(\bar{x}, \bar{y})$ according as $\sigma(i)=0$ or $\sigma(i)=1$.

(b) A formula $\varphi(\bar{x}, \bar{y})$ has the independence property for $T$ if for every $n \geqq 1, T$ implies the sentence.

$$
\psi^{n}: \exists \bar{w}_{0}, \cdots, \exists \bar{w}_{n-1} \underset{\sigma \varepsilon 2^{n}}{\wedge} \exists v_{\sigma} \underset{i<n}{\wedge} \psi\left(v_{\sigma}, \bar{w}_{i}\right)^{\alpha(i)}
$$

Thus a formula $\varphi(\bar{x}, \bar{y})$ is unstable in $T$ if for every $n$, a binary tree of length $n$ can be found where each node represents an instance $\varphi(x, \bar{a})$ of $\varphi(x, \bar{y})$ or a negation $\sim \varphi(x, \bar{a})$ and each path through the tree is satisfiable. The formula $\varphi$ has the independence property if this tree can be chosen so that all nodes at the same depth on the tree are either $\varphi(x, \bar{a})$ or $\sim \varphi(x, \bar{a})$ for the same $\bar{a}$.

The following examples illustrate some of the definitions of the last few paragraphs. The theory of algebraically closed fields is totally transcendental. For, if $F$ is an algebraically closed field, $S(F)$ consists of the types of elements of $F$ and the unique type of an element which is algebraically independent over $F$. The theory, $T h(Z,+)$, of the integers under addition is an example of a superstable but not totally transcendental theory. That is, for any model $B$ of $T h(Z,+)$, a member $p$ of $S(B)$ is determined by whether $p$ describes a member of $B$ or, if not, by which standard prime numbers divide each element which realizes $p$. Thus, $|S(B)|=|B|+2^{\boldsymbol{x}_{0}}$. If $T$ is an unstable theory then (Shelah (1971) a); §4) either some formula $\varphi(\bar{x}, \bar{y})$ has the strict order property (i.e. there is a model $A$ of $T$ and a sequence of sequences $\left\langle\bar{a}_{i}\right\rangle_{i<\omega}$ such that $A \mid=\exists \bar{x}\left(\varphi\left(\bar{x}, \bar{a}_{k}\right) \wedge \varphi\left(\bar{x}, \bar{a}_{l}\right)\right.$ if and only if $\left.k<l\right)$ or some formula $\psi(\bar{x}, \bar{y})$ has the independence property (i.e. there is a model $A$ and a sequence of sequences $\left\langle\bar{a}_{i}\right\rangle_{i<\omega}$ such that sets defined by the formulas $\varphi\left(\bar{v}, \bar{a}_{i}\right)$ are independent subsets of $A^{n}$ in the sense that any Boolean combination of these sets in nonempty.) In the presence of $G C H$, ded $(\kappa)=2^{\kappa}$ so the classes iv), v), and vi) defined above coalesce. But the syntactic distinction indicated in this paragraph remains. To see that in either case $T$ is unstable in the sense of the earlier definition note that in either case we can for any cardinal $\lambda$ by the compactness theorem expand the set $\left\langle\bar{a}_{i}\right\rangle_{i<\omega}$ to a set $X$ of cardinal $\lambda$. Moreover in the first case (compactness again) we may assume $X$ is densely linearly ordered. Now, for every cardinal $\lambda$, there is a dense linear order $X$ of power $\lambda$ with $\mid$ the set of Dedekind cuts of $X|>| X \mid$ (cf. Shelah (1971 a). Lemma 2.3). For this $X$, $|S(X)|>|X|=\lambda$. In the second case for any subset $Y$ of $X$ there is a member $p$ of $S(X)$ with $\varphi\left(v_{0}, \bar{x}\right) \in p$ if and only if $\bar{x} \in Y$. Thus $|S(X)| \geqq 2^{|x|}=2^{\lambda}$. As Shelah ((1971a): Theorem 4.7) points out, the theory of dense linear order without endpoints does not have the independence property. Numerous examples of theories with the independence property are given in Section 2. 
THEOREM 1.1 (Shelah (1971a)). (a) The theory $T$ is unstable if and only if some formula $\varphi(x, \bar{y})$ is unstable in $T$.

(b) The theory Thas the independence property if and only if some formula $\varphi(x, \bar{y})$ has the independence property in $T$.

We now apply these results to groups and obtain some simpler sufficient conditions for non-stability.

Definition. A subgroup $B$ of a group $A$ is definable in $A$ if there is a formula $\psi\left(v_{0}, \bar{a}\right)$ such that $B=\{b|A|=\psi(b, \bar{a})\}$, where $\bar{a}$ is a sequence of elements of $A$.

THEOREM 1.2. (a) If there is a sequence of definable subgroups $\left\langle B_{i}\right\rangle_{i<\omega}$ of $A$ with $B_{i} \supseteq B_{i+1}$ then $A$ is not totally transcendental.

(b) If, in addition, the index of $B_{i+1}$ in $B_{i}$ is infinite then $T$ is not superstable.

Proof. (a) Choose a sequence $\left\langle b_{i}\right\rangle$ with $b_{i} \in B_{i}-B_{i+1}$.

Then for each $k$, the translates of $B_{k}$ by appropriate products of the $\left\langle b_{i}\right\rangle_{i s k}$ produce $2^{k}$ disjoint infinite definable subsets of $A$. Thus there will be $2^{\boldsymbol{\alpha}_{1}}$ distinct types over a countably subset of $A$ consisting of $b_{1}, b_{2}, \cdots$ and the countably many constants necessary to define the $B_{i}$.

(b). If the index of $B_{i+1}$ in $B_{i}$ is infinite, we can, by the compactness theorem, find for any cardinal $\kappa$ and all $i$ simultaneously, a model of $T h(A)$ and elements $\left\langle b_{\alpha}^{i}\right\rangle_{\alpha<\kappa}$ such that $b_{\alpha}^{i} B_{i} \cap b_{\beta}^{i} B_{i}=\varnothing$ if $\alpha \neq \beta$. If we choose $\kappa$ with $\kappa^{\kappa_{0}}>\kappa$ we see $A$ is not superstable.

Theorem 1.2 depends strongly on the fact we are dealing with groups (or at least some class of algebras). There certainly exist totally transcendental theories with infinite decreasing sequences of definable sets. The key to Theorem 1.2 is the ability to translate subgroups. In fact, the theorem does not go through for ascending chains of definable subgroups. For, Sabbagh has pointed out that theory of the additive Abelian group $Z_{p^{*}}$ is $\omega$-stable (indeed $\boldsymbol{N}_{1}$-categorical) but contains an increasing chain of definable subgroups $B_{n}=$ $\left\{x \mid p^{n} x=0\right\}$. On the other hand if the $B_{n}{ }^{\prime} s$ are defined uniformly (see below) then $A$ is unstable and this result has nothing to do with group theory.

A sequence of subsets $B_{1}, B_{2} \cdots$ of a structure is uniformly definable if there is a formula $\varphi(x, \bar{y})$ and a sequence of sequences $\left\langle\bar{a}_{i}\right\rangle_{i<\omega}$ such that $B_{i}=\left\{b|A|=\varphi\left(b, \bar{a}_{i}\right)\right\}$.

THEOREM 1.3 (Shelah (1971) a)). If a group A contains an infinite increas ing (or decreasing) sequence of uniformly definable subsets (in particular subgroups) then $T$ is unstable.

Proof. This is just a special case of (Shelah 1971 a: Theorem 2.13). 
We relate one further method of proving unstability which hclds in general but is particularly useful in this context.

THEOREM 1.4. If $A \subseteq B$ and the quantifier free formula $\varphi(x, \bar{y})$ is unstable in $T h(A)$ (has the independence property in $T h(A)$ ) then $\varphi(x, \bar{y})$ is unstable (has the independence property) in $T h(B)$.

Proof. Note that the sentences $\varphi_{n}$ which asserts that $\varphi(x, \bar{y})$ is unstable in $T h(A)$ are all logically equivalent to existential sentences. So each $\varphi_{n}$ is true in $B$ and thus $\varphi$ is unstable in $\operatorname{Th}(B)$. The situation is exactly the same if $\varphi$ has the independence property.

We now relate one result which enables one to prove a group is stable.

THEOREM 1.5 (Macintyre (1970)). Let $A$ and $B$ be groups then the stability class of $A \oplus B$ is the same as the stability class of the less stable of $A$ and $B$. In particular, if $A$ and $B$ are totally transcendental (superstable, stable etc.) so is $A \oplus B$.

Proof. Macintyre proved that $|S(A \oplus B)|=|S(A)|+|S(B)|$. See also the introduction to Eklof and Fisher (1972).

\section{Section 2}

In this section we investigate the stability of various groups. We first report earlier work completely solving the problem for Abelian groups. We then make use of the results proved in Section 1 to prove various well-known groups are unstable.

The investigation of the stability classes of Abelian groups was begun by Macintyre (1970) who determined all the totally transcendental complete theories of Abelian groups. Berthier (1973) proved that every complete theory of Abelian groups is stable. The complete theories of Abelian groups were first determined by Szmielew (1955) and later by Elkof and Fisher (1972). In their paper Elkof and Fisher also established in which cardinals $\kappa$ a complete theory of Abelian groups has a saturated model.(See Sacks (1972) or the introduction to Eklof and Fisher (1972) for a definition.) It follows from work of Shelah (1971 a) that a countable complete theory $T$ is stable in $\kappa$ if and only if $T$ has a saturated model of power $\kappa$. One can thus determine from the table on page 146 of Eklof and Fisher (1972):

Theorem 2.1 (Shelah, Eklof, Fisher).

1. Every Abelian group is stable.

2. There are stable but not superstable Abelian groups.

3. There are superstable but not totally transcendental Abelian groups.

4. Every $\boldsymbol{N}_{0}$-categorical Abelian group is totally transcendental. 
The following example illuminates the situation somewhat. Let $T$ be the complete theory of Abelian groups which asserts $G$ is torsion-free and for every $p$ the dimension of $G / p G$ is infinite. (The completeness of $T$ is a special case of Szmielew's theorem.) Now the subgroups $n ! G$ form a decreasing sequence of subgroups $B_{n}$ such that $B_{n+1}$ is of infinite index in $B_{n}$. Hence, by Theorem $1.2 \mathrm{~b}$ ), $G$ is not superstable.

By taking the direct sum of a finite non-Abelian group with an Abelian group of the appropriate sort and applying Theorem 1, we see there are stable but not superstable, superstable but not totally transcendental, and also totally transcendental non-Abelian groups.

We now prove that various infinite permutation groups are unstable, indeed, have the independence property.

The symmetric group on $\kappa$ elements, $S_{\kappa}$, consists of all permutations of a set of $\kappa$ elements. The restricted symmetric group $S_{\kappa}^{\prime}$ consists of those permutations which move only finitely many elements.

The groups $S_{\kappa}$ give rise to various complete theories (depending on $\kappa$ ) whose properties are explored in McKenzie (1971). In contrast, it is easy to show by the method of Ehrenfeucht games that for any pair of infinite cardinals $\alpha$ and $\beta, S_{\alpha}^{\prime}$ is elementarily equivalent to $S_{\beta}^{\prime}$.

Lemma 2.2 The restricted Symmetric group $S_{\omega}^{\prime}$ has the independence property.

Proof. We show the formula $[x, y]=1$ is unstable in $S_{\omega}^{\prime}$. For fixed $n$, we must show $S_{\omega} \mid=\varphi^{n}$ where $\varphi^{n}$ is the formula in the definition of the independence property.

We choose elements $a_{0}, \cdots, a_{n-1}$ and $\left\langle b_{\sigma}\right\rangle \sigma \in 2^{n}$ to satisfy $\varphi^{n}$. Let $a_{i}$ be the transposition $(2 i, 2 i+1)$. Let $b_{s}$ be the product of the transposition $(3 n, 3 n+1)$ and the transpositions $(2 i, 4 n+i)$ for those $i$ such that $\sigma(i)=1$. Then $b_{\sigma}$ commutes with $a_{i}$ if and only if $\sigma(i)=0$, so $S_{\omega}^{\prime} \mid=\varphi^{n}$.

THEOREM 2.3. If $G$ contains the restricted symmetric group on an infinite set then $G$ has the independence property. In particular, for all infinite cardinals $\kappa, S_{\kappa}$ and $S_{\kappa}^{\prime}$ have the independence property.

Proof. The result is immediate from Lemma 2.2 and Theorem 1.4.

A group $G$ is algebraically closed if every set of equations with parameters from $G$ which is solvable in an extension of $G$ is solvable in $G$ (see Scott (1951). The following theorem answers a question raised by C. F. Miller and P. M. Schupp.

THEOREM 2.4. If $G$ is an algebraically closed group then $G$ has the independence property. 
Proof. It is well known that an algebraically closed group is in fact existentially closed (i.e. if $\varphi$ is an existential sentence with parameters in $G$ and $G \subseteq H$ where $H \mid=\varphi$ then $G \mid=\varphi$ ). Moreover, if $A$ and $B$ are groups there is a common extension of $A$ and $B$. Thus, given an algebraically closed group $C$ every finite group can be imbedded in $C$. (This construction occurs in Scott (1951)). But, slightly varying the construction of Theorem 2.3 we see that any group which contains the symmetric group on $n$-elements, for every finite $n$, has the independence property.

\section{Section 3}

In this section we give an application of the notion of stability to prove a theorem of a more traditionally group-theoretic nature.

In the following $Z(G)$ denotes the center of a group $G$ and $C_{G}(a)$ denotes the centralizer in $G$ of the element $a$. A group $G$ is locally nilpotent if every finitely generated subgroup of $G$ is nilpotent.

Lemma 3.1. Let $G$ be a locally nilpotent group such that for each $a \in$ $G \backslash Z(G), C_{G}(a)$ is $2 n$-1-step solvable then $G$ is $2 n+1$-step solvable.

Proof. Note, first, that it suffices to show $\bar{G}=G / Z(G)$ is $2 n$-step solvable. Now, suppose that for each $\bar{X} \neq 1 \in \bar{G}, C_{\bar{G}}(\bar{X})$ is $2 n$-step solvable. Then if $H$ is any finitely generated subgroup of $\bar{G}, H$ has a non-trivial center since $G$ is locally nilpotent. Thus $H \subseteq C_{\tilde{G}}(\bar{X})$ for some $\bar{X} \neq 0$ so $H$ is $2 n$-step solvable and so $\bar{G}$ is $2 n$-step solvable. Now for a fixed $\bar{X} \neq 1$, let $\bar{C}=C_{\bar{\sigma}}(\bar{X})$ and let $C$ be the inverse image of $\bar{C}$ in $G$. We show that $C$ is $2 n$-step solvable by noticing that if $x$ is a preimage of $\bar{X}$, then $C^{\prime}$, the derived group of $C$, is contained in $C_{G}(x)$ which is $2 n$-1-step solvable by hypothesis. Let $f, g \in C$. Then $x^{f}, X^{g}$ are in $x Z(G)$ so $x^{f}=x b$ and $x^{8}=x c$ with $b, c \in Z(G)$. Hence, $x^{f 8}=x b^{8}=x b c=$ $x c b=x c^{g}=x^{g f}$. Thus $[f, g] \in C_{G}(x)$ as desired.

Lemma 3.2 Let $G$ be a locally nilpotent group. For every natural number $n$ either:

i) $G$ is 2n-1-step solvable

or ii) There exist $a_{1}, \cdots, a_{n} \in G$ such that for each $i \leqq n$,

$$
a_{i} \in \bigcap_{i<j} C_{G}\left(a_{j}\right) \backslash Z\left(\cap_{j<i} C_{G}\left(a_{i}\right)\right) \text {. }
$$

Proof. The proof is by induction on $n$. If $n=1$ the result is trivial. Suppose now that $n>1$ and we have proved the result for $n-1$. Either, we can choose $a_{1}, \cdots a_{n}$ to satisfy i) or for each $a_{1} \in G \backslash Z(G), C_{G}\left(a_{1}\right)$ is $2 n$-step solvable by induction hypothesis. But then by Lemma $3.1 G$ is $2 n$-1-step solvable as desired.

Now we can give the promised application of stability. 
THEOREM 3.2. If a locally nilpotent group $G$ is stable then $G$ is solvable.

Proof. We suppose that $G$ is not solvable and invoke ii) of Lemma 3.2. We will rely on (Shelah $1971 \mathrm{a}$ : $\$ 2$ ): which implies that a formula $\varphi(x, y)$ is unstable if for each $n$ there exist $a_{1}, \cdots, a_{n}, b_{1}, \cdots, b_{n}$ such that $\varphi\left(a_{i}, b_{i}\right)$ holds if and only if $i<j$, to show the formula $[x, y]=1$ is unstable in $G$. (In fact, we show $[x, y] \neq 1$ has the order property (Shelah 1971 a: Definition 2.2)). Let $a_{1}, \cdots, a_{n}$ be as in Lemma 3.2 ii). We proceed by a double induction to choose appropriate $b_{n}, b_{n-1}, \cdots, b_{1}$. Suppose $b_{n}, \cdots, b_{n-(k-1)}$ have been chosen. Since

$$
a_{n-k} \in \bigcap_{i<n-k} C_{G}\left(a_{i}\right) \backslash Z\left(\bigcap_{i<n-k} C_{G}\left(a_{i}\right)\right),
$$

there is an element $b_{n-k}^{*}$, such that $\left[b_{n-k}^{*}, a_{i}\right]=1$ for $i<n-k$ and $\left[b_{n-k}^{*}, a_{n-k}\right] \neq 1$. We now choose $c_{0}=b_{n-k}^{*}, c_{1}, \cdots, c_{k}=b_{n-k}$ such that $\left[c_{i}, a_{j}\right]=$ 1 if $j<n-k$ and $\left[c_{l}, a_{j}\right] \neq 1$ if $n-k \leqq j \leqq n-k+l$. Suppose we have chosen such a $c_{m}$. If $\left[c_{m}, a_{n-k+m+1}\right] \neq 1$ let $c_{m+1}=c_{m}$. If not, let $c_{m+1}=c_{m} b_{n-k+m+1}$. To see $c_{m+1}$ is an appropriate choice recall that if $[x, y]=1,[x, z] \neq 1$ then $[x, y z] \neq 1$. Thus we choose elements $b_{n}, \cdots, b_{1}$ such that $\left[a_{i}, b_{k}\right]=1$ if and only if $j<k$ so $G$ is unstable as desired.

This theorem naturally raises the question of whether there are other group theoretic conditions which in conjunction with stability might imply that a group is solvable. Sabbagh (1975 b) has shown that if $K$ is an algebraically closed field and $n>1$ then PSL $(n, k)$ is a totally transcendental infinite, simple group so some further condition is necessary.

The results of the next section, (see also Sabbagh (1975 a, b)), show that there are many nilpotent unstable groups. The following concrete example shows that, unlike direct product (Theorem 1.5), semidirect product does not preserve stability.

Let $A$ be the direct sum of countably many copies of $Z_{3}$ with generators $a_{0}, a_{1}, \cdots$, and let $B$ the direct sum of countably many copies of $Z_{3}$ with generators $b_{0}, b_{1}, b_{2}, \cdots$, and let $\theta: B \rightarrow$ Aut (A) define the action of $B$ on $A$ as follows

$$
\theta\left(b_{i}\right)\left(a_{j}\right)=\left\{\begin{array}{lll}
a_{i} & \text { if } & j \leqq i \\
a_{j} a_{0} & \text { if } & j>i
\end{array}\right\} .
$$

Then the semidirect product $A X_{\theta} B$ is nilpotent of class 2 but unstable. This example arose out of discussions with Peter Neumann.

\section{Section 4}

The definitions given in Section 1 dealt with complete theories. We can extend our investigation to incomplete theories as follows. 
Definition. A theory $T$ is stable (superstable etc.) if and only if every complete extension of $T$ is stable (superstable etc.).

This definition accords with the definition of stability for incomplete theories given in Berthier (1973).

A variety of groups is a class of groups defined by a set of equations. Birknoff's theorem asserts that a class $K$ of groups is a variety if and only if $K$ is closed under homomorphism, subalgebra and direct product.

As remarked in Section 2 every complete theory and thus, in particular, every variety of Abelian groups is stable. In contrast, we have the following result which relates to a question of Keisler. See also: Sabbagh (1975 b).

THEOREM 4.1. Every non-Abelian variety of groups is unstable and, in fact, has the independence property.

Proof. Let $K$ be any non-Abelian variety of groups and let $A$ be a non-Abelian member of $K$. Choose $a, b \in A$ such that $a$ and $b$ do not commute. Let $B$ be $A^{\omega}$ (the direct product of countably many copies of $A$ ). Let $a_{n}, n \in \omega$ and $d_{S}, S \subseteq \omega$, be the following elements of $A^{\omega}$ :

$$
\begin{aligned}
& a_{n}(i)=\left\{\begin{array}{lll}
e & \text { if } & i \neq n \\
a & \text { if } & i=n
\end{array}\right. \\
& d_{S}(i)=\left\{\begin{array}{lll}
e & \text { if } & i \in S \\
b & \text { if } & i \notin S
\end{array}\right.
\end{aligned}
$$

Then $d_{s}$ commutes with $a_{n}$ if and only if $n \in S$. It is now easy to see that the formula $[x, y]=1$ ( $x$ commutes with $y$ ) has the independence property in $T h(B)$ and hence, by Shelah (1971 a), Th $(B)$ and thus $K$ has the independence property.

Our proof also yields (cf. Baldwin and Lachlan 1973).

COROLlary 1. If $T$ is a complete theory of groups such that

i) Thas a non-Abelian model and

i) the class of models of $T$ is closed under direct product then $T$ is unstable. Indeed, $T$ has the independence property.

At the AMS colloquium lectures (1975), see Keisler (1975), Keisler asked whether there was any extension of the theory of groups which was neither $\aleph_{0}$-stable nor had the independence property for quantifier free types. We have shown here that if $T$ is any extension of the theory of groups satisfying: (i) $T$ has a non-Abelian model and $T$ is closed under direct product then there is a quantifier free formula of $T$ which has the independence property.

We ask, is there a group $G$ such that the universal theory of $G$ (the set of all sentences true in $G$ which have only universal quantifiers) has an unstable 
quantifier-free formula, but no quantifier-free formula has the independence property?

In the original draft of this paper we asked whether an unstable group i.e. the complete theory of $G$ unstable, must have the independence property. Sabbagh has pointed out that if $k$ is a real closed field then it is implicit in Sabbagh (1975 b),.(using the results of Malcev referred to there), that $G L(n, k)$ is unstable, but $G L(n, k)$ does not have the independence property.

The following question which is suggested by Lachlan's (1974) question of whether an $\boldsymbol{N}_{0}$-categorical stable theory must be totally transcendental remains open.

Does there exist a stable but not totally transcendental group of bounded exponent?

\section{References}

J. T. Baldwin and A. H. Lachlan (1973), 'On universal Horn classes categorical in some infinite power', Algebra Universalis, 3, 98-111.

D. Berthier (1973), 'Stability, Products, Groups', Notices Amer. Math. Soc. 73T-E98.

P. Eklof and E. Fisher (1972), 'The elementary theory of Abelian groups', Annals Math. Log. 4, 115-171.

H. J. Keisler (1974), 'The number of types in a first order theory', Notices Amer. Math. Soc. 74T-E8 p. A316. Keisler 1975.

H. J. Keisler (1975), 'Six Classes of Theories', J. Austral. Math. Soc. 21 (Series A), 129-138.

A. H. Lachlan (1974), 'Two conjectures regarding $\omega$-stable theories', Fund. Math. 81, 133-145.

A. Macintyre (1970), 'On $\omega_{1}$-categorical theories of Abelian groups', Fund. Math. 70, $253-270$.

R. McKenzie (1970), 'On elementary types of symmetric groups', Alg. Univ. 1, 13-21.

M. Morley (1965), 'Categoricity in power', Trans. Amer. Math. Soc. 114, 514-538.

J. G. Rosenstein (1973), ' $\boldsymbol{N}_{0}$-categoricity of Groups', $J$. of Algebra 25, 435-467.

G. Sabbagh (1975a), 'Logique Mathematique. - Categoricité et Stabilité: construction les préservant et conditions de chaine', C. R. Acad. Sc. Paris, t. 280 (3 Mars 1975).

G. Sabbagh (1975b), 'Logique Mathematique. - Categoricité et stabilité: quelques examples parmi les groupes et anneaux', C. R. Acad. Sc. Paris, t. 280 (10 Mars 1975).

G. E. Sacks (1972), 'Saturated Model Theory', W. A. Benjamin Inc., Reading, Mass.

W. R. Scott (1951), 'Algebraically Closed Groups', Proc. Amer. Math. Soc. 2, 118-121.

S. Shelah (1969), 'Stable theories', Israel J. Math. 7, 187-202.

S. Shelah (1971a), 'Stability, the f.c.p., and superstability; model theoretic properties of formulas in first order theory', Annals Math. Log. 3, 271-362.

S. Shelah (1971b), 'The number of non-isomorphic models of an unstable first-order theory', Israel $J$. Math., 9, 473-487.

W. Szmielew (1955), 'Elementary properties of Abelian groups', Fund. Math. 41, 203-271.

Department of Mathematics

University of Illinois at Chicago Circle

Box 4348

Chicago, Illinois 60680

U.S.A. 